\title{
ON THE SOLUTION OF SOME SIMPLE FRACTIONAL DIFFERENTIAL EQUATIONS
}

\author{
L.M.B.C. CAMPOS \\ Instituto Superior Tecnico \\ 1096 Lisboa Codex \\ Portuga 1 \\ (Received May 12, 1988)
}

\begin{abstract}
The differintegration or fractional derivative of complex order $\nu$, is a generalization of the ordinary concept of derivative of order $n$, from positive integer $v=n$ to complex values of $v$, including also, for $v=-n$ a negative integer, the ordinary n-th primitive. Substituting, in an ordinary differential equation, derivatives of integer order by derivatives of non-integer order, leads to a fractional differential equation, which is generallya integro-differential equation. We present simple methods of solution of some classes of fractional differential equations, namely those with constant coefficients (standard I) and those with power type coefficients with exponents equal to the orders of differintegration (standard II). The fractional differential equations of standard I (II), both homogeneuus, and inhomogeneous with exponential (power-type) forcing, can be solved in the 'Liouville' ('Riemann') systems of differintegration. The standard I (II) is linear with constant (non-constant) coefficients, and some results are also given for a class of non-1inear fractional differential equations (standard III).
\end{abstract}

KEY WORDS AND PHRASES. Fractional Derivatives and Fractional Differential Equations 1980 AMS SUBJECT CLASSIFICATION CODE. 34A

§1. INTRODUCTION.

The differintegration operator (Ross [1], 01dham and Spanier [2], Lavoie and Tremblay and Osler [3], McBride [4], Nishimoto [5], Campos [6], McBride and Roach [7] may be interpreted as a derivative (or integral) of complex order $+v$ (or $-v$ ), which reduces to the ordinary $n$-th derivative (primitive) for $\nu=+n(\nu=-n)$ a positive (negative) integer. Differintegration operators have found applications in such diverse fields as the hydraulics of dams (Ross [1]), potential fields (Weinstein [8]; Erdelyi [9, 10], [12]) diffusion problems (01dham and Spanier [11]) and waves in liquids (Lighthill [12]) and gases (Campos [13]). In some applications, the solution can be conveniently expressed in terms of fractional derivatives of elementary functions, e.g. In the scattering of acoustic waves (Marston [14]) or vibrations of visco-elastic rods (Campos [15]). Fractional derivatives have been used to solve ordinary (Nishimoto [5]) and partial (01dham and Spanier [16]) differential equations. A more general problem is the solution of fractional differential equations (F.D.E.s), obtained by replacing in ordinary differential equations (O.D.E.s), some or all of the ordinary derivatives of complex order. 
Although the subject of fractional differential equations is old (Liouville [17, 18, 19]), it does not appear to have been considered systematically in the modern literature; several of the recent applications mentioned above involve relation(s) between a function and its differintegration(s), which are particular instances of F.D.E.s. The applications of F.D.E.s are potentially wider than the problems mentioned above in connection with the fractional calculus, because the fractional differential equations provide generalizations of the integral equations of Abel's (Sneddon [20]) and Volterra's [21] types, which occur in many problems. A specific F.D.E. representing an harmonic oscillator with memory-type damping, has been considered in seismic problems (Duarte [22]), and solved using Fourier analysis (Duarte [23]); the latter equation is a particular instance of a linear fractional differential equation with constant coefficients, and this class of F.D.E.s can be solved readily using methods similar to those applied to O.D.E.s (Forsyth [24]), provided that the rules of differintegration (Lavoie and Tremblay and 0sler [3]; Campos [25]) can be used. The implication is that certain classes of fractional differential equations, which are actually integro-differential equations generalizing Abel's and Volterra's types, can be solved as simply as 0.D.E.s as will be shown in the present paper.

Two classes of fractional differential equations, which as mentioned in the introduction $(\$ 1)$ can be solved by very simple methods akin to those ir the theory of O.D.E.s are the linear equation with constant coefficients (standard I), and with power type coefficients with exponent equal to the order of differintegration (standard II). Concerning the former (Part 1), we start by considering a simple case, to address the following issue: (\$3) given that an O.D.E. of order $N$ has $N$ linearly independent particular integrals (L.I.P.I), how many L.I.P.I. has a F.D.E.?; We proceed to solve the general linear F.D.E. of Standard I, in the homogeneous case (\$4), and in the inhomogeneous case with exponential forcing (\$5), concluding with an example ( $\$ 8)$ of a forced oscillator with memory-type damping. Concerning the F.D.E. of standard II we must use (Part II), the 'Riemann" system of differintegration, instead of the 'Liouville' system used (Part I) for standard I, the two systems being incompatible (Lavoie and Tremblay and Osler [3]; Campos [25]). The solution of the standard II is as straightforward as of standard I, both for the simplest (\$8) and most general ( $\$ 9$ ) homogenous F.D.E., and for the inhomogeneous F.D.E. with power-type forcing ( $(10)$; although all of the preceding F.D.E.s are linear, similar simple methods can be used to solve (\$11) a restricted class (standard III) of non-linear F.D.E.s.

PART I - INTEGRODIFFERENTIAL EQUATIONS OF THE GENERALIZED ABEL TYPE

§2. INTRODUCTION TO GENERALIZED ABLE EQUATIONS.

The general linear inhomogeneous fractional differential equation with constant coefficients $A_{m}$, and differintegrations of order $\nu_{m}$, is:

$$
\sum_{m=1}^{M} A_{m} D^{\nu_{m}} F / D z^{\nu_{m}}=G(z),
$$

where $G(z)$ is the forcing function, and $F(z)$ the solution. The 'Liouville' differintegration $D^{\nu} / D z^{\nu}$ of an analytic function $F(z)$ is given by: (1) for complex order with negative real parts (Campos [6]): 


$$
\operatorname{Re}(\nu)<0: D^{\nu} F / D z^{\nu}=\{\Gamma(-\nu)\}^{-1} \int_{\infty}^{z}(z-x)^{-\nu-1} F(x) d x
$$

viz, for $\nu=-n$ a negative integer, $(2.2)$ is the $n-t i m e s$ repeated integral from $\infty$ to z; (ii) for complex order other than a negative integer (Nishimoto [5]):

$$
\operatorname{Re}(\nu) \neq-1,-2, \ldots: D^{\nu} F / D z^{\nu}=\{\Gamma(1+\nu) / 2 \pi i\} \int_{\infty}^{(z+)} \exp (i \arg z)(\zeta-z)^{-\nu-1} F(\zeta) d \zeta,(2.3)
$$

viz, for $\nu=n$ a positive integer the (Hankel [26]) path of integration can be closed to a loop around $\zeta=z$, and (2.3) reduces to Cauchy's theorem for the ordinary n-th derivative. Thus $(2.1)$ is an ordinary integrodifferential equation if all orders $\nu_{1}, \ldots, v_{M}$ are integers, whereas if some orders are non-integral, it has terms of the type (2.2) or (2.3), justifying the designation of generalized Abel type.

\section{NUMBER OF LINEARLY INDEPENDENT INTEGRALS.}

The original Abel's equation (Sneddon [20]) is a particular homogeneous sub-case of (2.1), with $G(z)=0=\nu_{1}, \nu_{2} \equiv \nu, M=2, \lambda \equiv A_{1} / A_{2}, v i z$. :

$$
D^{\nu} F / D z^{\nu}-\lambda F(z)=0
$$

where the integral in (2.2) and (2.3) is indefinite. If $v=n$ is a positive integer, then (3.1) is an ordinary differential equation (0.D.E.) with $n$ linearly independent particular integrals (L.I.P.I.S); we may thus enquire about the number of L.I.P.I.S of (3.1) when $\nu$ is not an integer. The answer to this question will be obvious, once (3.1) is solved. The equation (3.1) can be solved in any 'Liouville' type [17, 27] system of differintegration, in which:

$$
D^{\nu}\left\{e^{a z}\right\} / D z^{\nu}=a^{\nu} e^{a z},
$$

holds, e.g. this is the case (Campos [25]) for (2.2) and (2.3).

We may seek a solution of (3.1) in the form (3.3a):

$$
F(z)=e^{a z}, \lambda=a^{\nu}
$$

where the constant a satisfies (3.3b); the latter is an algebraic equation with roots $a_{k}$ given by:

$$
\log a_{k}=(\log \lambda) / \nu=(\log \lambda) / \nu+k 2 \pi i / v
$$

where Log denotes the principal branch of the logarithm, and the integer $k$ identifies all other branches, each leading to one value $a_{k}$ of $a$. To each value $a_{k}(3.4)$ corresponds a particular integral (3.3a), viz.:

$$
F_{k}(z)=\exp \left(a_{\alpha} z\right)=\exp \left\{\lambda^{1 / \nu} e^{k 2 \pi i / \nu_{z}}\right\}
$$

where $\lambda^{1 / \nu}$ denotes the principal branch of the complex root. The number of linearly independent particular integrals (3.5) of (3.1), depends on $\nu$, viz.: (i) if $\nu=n$ is a 
positive integer, there are $n$ L.I.P.I., e.g. (3.5) with $k=0, \ldots, n-1, v i z .(3.1)$ in this case is an 0.D.E.; (ii) if $\nu=p / q$ is a rational number, there are p L.I.P.I., e.g. (3.5) with $k=0, \ldots, p$; (iii) if $\nu$ is a real irrational number, or is a complex number, the particular integrals (3.5) are linearly independent for all integer $k=0$, $\pm 1, \pm 2, \ldots, \pm \infty, 1 . e$. they form a denumerably infinite set.

The L.I.P.I. (3.5) of the F.D.E. (3.1) can be denoted by $F_{k}(z)$, with $k=\alpha$, $\ldots, \beta$, and $\alpha=0, \beta=n$ in the case (i) of $\nu=n$ a positive integer, $\alpha=0, \beta=p$ in the case (ii) of $\nu=p / q$ a rational number, and $\alpha=-\infty, \beta=+\infty$ in the case of $\nu$ a real irrational or complex number. The general integral of the F.D.E. is a linear combination of the L.I.P.I.S (3.5):

$$
F(z)=\sum_{k=\alpha}^{\beta} C_{k} \quad F_{k}(z)=\sum_{k=\alpha}^{\beta} C_{k} \exp \left\{\lambda^{1 / \nu} e^{k 2 \pi i / \nu} z\right\},
$$

where $C_{k}$ are abitrary constants. An example of the solution of the F.D.E. (3.1) with $\nu=i, \lambda=1$ is

$$
D^{i} F / D z^{i}=F(z) ; F(z)=\sum_{k=-\infty}^{+\infty} C_{k} \exp \left\{e^{2 \pi k} z\right\},
$$

where the 'constants of integration' $C_{k}$ may be restricted so that the solution converges, e.g. for $z \equiv x$ real. For example, if $x>0$, the general term of the series (3.7) diverges $\exp \left\{e^{k 2 \pi} x\right\}+\infty$ as $k+\infty$, unless the coefficients $c_{k}=0$ vanish, beyond a certain order $k>m$; the latter is thus a necessary condition for convergence. If $x$ $<0$, then $\exp \left\{e^{k 2 \pi} x\right\}<1$ for all $k$, and a sufficient condition for the convergence of the series ( 3.7$)$, is that the series of coefficients $\sum c_{k}$ converges for $k=-\infty, \ldots,+\infty$.

\$4. LINEAR, HOMOGENEOUS F.D.E. WITH CONSTANT COEFFICIENTS.

The general linear F.D.E. with constant coefficients (2.1), in the homogeneous case $G(z)$, consists of a polynomial $P(D)$ of derivatives of complex order:

$$
\{P(D)\} F(z) \equiv \sum_{m=1}^{M} A_{m} D^{\nu_{m}} F / D z{ }^{\nu_{m}}=0,
$$

applied to the function $F(z)$. Using the 'Liouville' rule (3.2), the solution of (4.1) exists in the form of an integral function (3.3a), provided that a be a root of

$$
F(z)=e^{a z} ; 0=P(a)=\sum_{m=1}^{M} A_{m} a^{\nu_{m}}
$$

the characteristic pseudopolynomial (4.2), which is a polynomial of powers with complex exponents equal to the orders of differintegration in the F.D.E. (4.1), and having the same coefficients. If $a_{k}$ with $k=\alpha, \ldots, \beta$ denote all the roots of (4.2), and if they are all distinct, and

$$
R\left(a_{k}\right) \neq 0 ; P(a)=R(a) \prod_{k=\alpha}^{\beta}\left(a-a_{k}\right),
$$

then the general integral of the F.D.E. (4.1) is a linear combination of the L.I.P.I. (3.3a) for each $a_{k}$, viz. 


$$
F(z)=\sum_{k=\alpha}^{\beta} C_{k} F_{k}(z)=\sum_{k=\alpha}^{\beta} C_{k} \exp \left(a_{k} z\right),
$$

where $C_{k}$ are constants.

In the case where $a_{k}$ is a root of multiplicity $s$ of the characteristic pseudopolynomial (4.2), and

$$
Q\left(a_{k}\right) \neq 0 ; P(a)=\left(a-a_{k}\right)^{s} Q(a)
$$

then $\exp \left(a_{k} z\right)$ is one particular integral of the F.D.E. (4.1), and we can find (s - 1) more L.I.P.I.s by the procedure that follows. The pseudopolynomial equation $(4.2)$, in the present case $(4.5)$ of root of multiplicity $s$,

$$
0=P(a) e^{a z}=Q(a)\left(a-a_{k}\right)^{s} e^{a z},
$$

is satisfied in the limit $a \rightarrow a_{k}$, even if we differentiate $\ell$ times with regard to a:

$$
0=\lim _{a \rightarrow a_{k}} \frac{\partial^{\ell}}{\partial a^{\ell}}\left\{P(a) e^{a z}\right\}=\lim _{a+a_{k}} \sum_{m=1}^{\ell}\left(\int_{m}^{l}\right) P^{(m)}(a) a^{l-m} e^{a z},
$$

because $P^{(l)}(a)=0$ for $l=0, \ldots, s-1$. The result (4.7) shows that if $a_{k}$ is a root of multiplicity $s$ of the pseudocharacteristic polynomial (4.5), to it corresponds $s$ particular integrals of the F.D.E. (4.1), namely

$$
F_{k}^{\ell}(z)=\lim _{a+a_{k}} \partial^{\ell}\left(e^{a z}\right) / \partial a^{\ell}=z^{\ell} \exp \left(a_{k} z\right) .
$$

The particular integrals (4.8) are linearly independent for distinct roots $a_{k}$, and for each root of multiplicity $s_{k}$, for distinct $\ell=0, \ldots, s_{k}-1$.

We have shown that, if the characteristic pseudopolynomial (4.2) has distinct roots $a_{k}$ with $k=\alpha, \ldots, \beta$, of multiplicities $s_{k}$,

$$
R\left(a_{k}\right) \neq 0 ; P(a)=R(a) \prod_{k=\alpha}^{B}\left(a-a_{k}\right)^{s_{k}},
$$

then the general integral of the F.D.E. (4.1) is a linear combination of the L.I.P.I.S $(4.8)$,

$$
F(z)=\sum_{k=\alpha}^{\beta} \sum_{\ell=0}^{s_{k}-1} c_{k}^{\ell} F_{k}^{\ell}(z)=\sum_{k=\alpha}^{\beta} \exp \left(a_{k} z\right) \sum_{\ell=0}^{s_{k}-1} c_{k}^{\ell} z^{\ell},
$$

where the $c_{k}^{l}$ are constants. For example, the linear homogeneous fractional differential equation

$$
D^{1} F / D z^{i}+D^{-1} F / D z^{-1}-2 F(z)=0
$$

has characteristic pseudopolynomial

$$
0=P(a)=a^{1}+a^{-1}-2=a^{-1}\left(a^{i}-1\right)^{2},
$$

with the same roots as (3.7), with the important distinction that all roots are double instead of simple; it follows that the general integral (3.7) is replaced by (4.10) with $s_{k}=2$, viz. 


$$
F(z)=\sum_{k=-\infty}^{+\infty}\left(C_{k}+B_{k} z\right) \exp \left\{e^{2 \pi k} z\right\}
$$

where $C_{k} \equiv C_{k}^{0}$ and $B_{k} \equiv C_{k}^{1}$ are constants.

§5. INHOMOgENEOUS EQUATION WITH EXPONENTIAL FORCING TERM.

The complete integral of the linear inhomogenous fractional differential equation (2.1), consists of the general integral of the homogenous equation (4.1), viz.

$$
F(z)=\sum_{k=\alpha}^{\beta} C_{k} F_{k}(z)+F_{o}(z),
$$

plus a particular integral $F_{0}(z)$ of the inhomogeneous equation. For example, if the forcing term is a complex exponential (5.2a)

$$
G(z)=B e^{b z}, F_{0}(z)=C_{\star} e^{b z}
$$

a particular integral (5.2b) is also a complex exponential, with coefficient $C_{\star}$ determined by

$$
B e^{b z}=\{P(D)\} C_{\star} e^{b z}=C_{\star} P(b) e^{b z}
$$

Assuming that $b$ is not a root of the pseudocharacteristic polynomial $P(b) \neq 0$, we can solve (5.3) for $C_{\star}=B / P(b)$, and obtain a particular integral (5.2b) of the inhomogeneous F.D.E. (2.1) with exponential forcing (5.2a), viz.

$$
P(b) \neq 0 ; F_{0}(z)=\{B / P(b)\} e^{b z},
$$

where $P(b)$ is defined by $(4.2)$.

If $b$ is a root of multiplicity $s$ of the pseudocharacteristic polynomial

$$
P(a)=(a-b)^{s} Q(a), P^{(s)}(b)=s ! Q(b) \neq 0
$$

we cannot divide $(5.3)$ by $P(b)$, to obtain $(5.4) \equiv(5.2 b)$. In this case, we start with the formal identity (5.3) with b replaced by $a$,

$$
B e^{a z}=C_{\star}(a-b)^{s} Q(a) e^{a z},
$$

and differentiate $s$ times with regard to a to obtain

$$
\text { B } z^{s} e^{a z}=C_{*} s ! Q(a) e^{a z}+O(a-b),
$$

before letting $a+b$ :

$$
\text { B } z^{s} e^{b z}=C_{\star} s ! Q(b) e^{b z}=s ! Q(b) F_{0}(z),
$$

where the particular integral $F_{0}(z)$ was substituted from (5.2b). Since $Q(b) \neq 0$ for $a$ root (5.5b) of multiplicity $s$, of the characteristic pseudopolynomial (5.5a), we can solve (5.9) for the particular integral 


$$
P(a) \sim O\left((a-b)^{s}\right) ; F_{0}(z)=\{B / s ! Q(b)\} z^{s} e^{b z}=\left\{B / P^{(s)}(b)\right\} z^{s} e^{b z},(5 \cdot 10)
$$

of the inhomogeneous F.D.E. (2.1), (4.2).

For example, the inhomogeneous linear fractional differential equation with constant coefficients and exponential forcing,

$$
D^{i} F / D z^{i}+D^{-i} F / D z^{-i}-2 F(z)=e^{b z},
$$

has characteristic pseudopolynomial (4.12),

$$
P(b)=b^{i}+b^{-i}-2=2(\cos \log b-1)
$$

Hence, a particular integral of (5.11) is (i) given by (5.4):

$$
\log b \neq 2 \pi k: F_{0}(z)=\frac{1}{2}\{\cos \log b-1\}^{-1} e^{b z} \text {, }
$$

provided that $b$ is not one of the roots $a_{k}=e^{k 2 \pi}$ associated with (4.13) of the homogeneous F.D.E. (4.11), i.e. $b \neq e^{2 \pi k}$ for all integer $k$; (ii) if $b=e^{2 \pi k}$ for some integer $k$, then the pseudocharacteristic polynomial (5.12) has a double root

$$
P^{\prime \prime}\left(e^{2 \pi k}\right)=-2 e^{-4 \pi k},
$$

and the particular integral is $(5.10)$ with $s=2$, viz.

$$
b=e^{2 \pi k}: F_{0}(z)=2 e^{-4 \pi k z^{2}} \exp \left\{e^{2 k \pi} z\right\}
$$

The complete integral of the inhomogenous F.D.E. (5.11) is obtained by adding (4.13) to the particular integral (5.13) or (5.15).

§6. OSCILLATION AND RESONANCE WITH NON-INTEGRAL DAMPING EXPONENT.

As a demonstration of the simplicity of the present method of approach to fractional differential equations, we reconsider a free or forced harmonic oscillator with memory-type damping, which was solved elsewhere (Duarte [22]), using Fourier analysis to study the free motion only. When a particle moves along a trajectory $x(t)$, defined by a coordinate $x$ as a function of time $t$, the viscous damping force is taken as proportional and opposite to the velocity

$$
0<r<2 ; s(t)=-\mu D^{r} x / D t^{r}
$$

where $r=1$ and $\mu$ is the damping coefficient. Other integer values of the exponent $r$ correspond to the inertia force associated with mass $m$ for $r=2$, and to a linear spring of constant $q$ for $r=0$; their combined motion under forcing with amplitude $f$ and frequency $\omega$, is specified by

$$
m D^{2} x / D t^{2}+\mu D^{r} x / D t^{r}+q x=f e^{i \omega t}
$$


The case of a friction force (5.16) with order of differintegration $0<r<2$ between an elastic $r=0$ and an inertia $r=2$ force, has been considered in connection with the modeling of seismic events (Duarte, [22]); whereas for the viscous damping, the force (5.16) with $r=1$ is a function of local velocity at the 'present' point $x(t)$, and for $r \neq 1$

$$
D^{r} x / D t^{r}=\left(D^{2} / D t^{2}\right)\{\Gamma(2-k)\}^{-1} \int_{-\infty}^{t}(t-\tau)^{k-2} x(\tau) d \tau
$$

it depends on the trajectory $x(\tau)$ for all past $t$ ime $t>\tau>-\infty$, introducing a memory effect (Volterra [21]).

We consider first the free motion, unafected by forcing $f=0$, so that we obtain the homogenous F.D.E. ( 4.1$)$, with characteristic pseudopolynomial

$$
P(a)=a^{2}+2 \lambda a^{r}+\omega_{0}^{2}
$$

where $\lambda$ is the temporal damping, and $\omega_{0}$ the natural frequency,

$$
\lambda=\mu /(2 \mathrm{~m}), \omega_{0}=\sqrt{\mathrm{q} / \mathrm{m}} \text {. }
$$

The roots $a_{k}$ of $P\left(a_{k}\right)=0$ specify (5.19) the particular integrals exp( $\left.a_{k} t\right)$, implying that: (i) the motion is oscillatory if $\operatorname{Im}\left(a_{k}\right) \neq 0$, and monotonic if $\operatorname{Im}\left(a_{k}\right)=0$; (ii) the amplitude is constant if $\operatorname{Re}\left(a_{k}\right)=0$, and increases with time for $\operatorname{Re}\left(a_{k}\right)>0$ and decreases with time for $\operatorname{Re}\left(a_{k}\right)<0$. Thus, the free motion consists of the superposition of modes

$$
x(t)=\sum_{k=\alpha}^{\beta} c_{k} \exp \left(a_{k} t\right)=\sum_{k=\alpha}^{\beta} c_{k} \exp \left\{\left(-\lambda_{k}+i u_{k}\right) t\right\},
$$

with frequency $w_{k}=\operatorname{Im}\left(a_{k}\right)$ and decay $\lambda_{k}=-\operatorname{Re}\left(a_{k}\right)$.

The forced motion, adds to (5.21), a particular solution of the inhomogenous F.D.E. (5.17) with $(5.20 \mathrm{ab})$, viz.

$$
D^{2} x / D t^{2}+2 \lambda D^{r} x / D t^{r}+\omega_{0}^{2} x(t)=(f / m) e^{i \omega t}
$$

The forced motion is specified by (5.4), viz.

$$
P(i \omega)=\omega_{0}^{2}-\omega^{2}+2 \lambda(i \omega)^{r} \neq 0 ; x(t)=(f / m)\{P(i \omega)\}^{-1} e^{i \omega t},
$$

if the forcing frequency $\omega$ is not a root of the characteristic pseudopolynomial, in which case the amplitude of the motion is a constant $(f / m) /|P(i \omega)|$, and there is a constant phase $1 \mathrm{ag} \arg \{\mathrm{P}(i \omega)\}$. If $\omega$ is a root of multiplicity $s$ of the characteristic pseudopolynomial, the forced motion is given by (5.10), viz.

$$
x_{0}(t)=(f / m)\left\{\partial^{s} P(i \omega) / \partial \omega^{s}\right\}^{-1} i^{-s} t^{s} e^{i \omega t}
$$

which corresponds to a resonance of order $s$, with amplitude growing in $t i m e t^{s}$ and a phase shift $-s \pi / 2$. 
PART II - INTEGRODIFFERENTIAL EQUATIONS OF THE GENERALIZED VOLTERRA TYPE

§7. INTRODUCTION TO GENERALIZED VOLTERRA'S EQUATION.

Another class (standard II) of linear fractional differential equation is

$$
\sum_{m=1}^{M} A_{m} z^{\nu_{m}} d^{\nu_{m}} F / d z{ }^{\nu_{m}}=G(z),
$$

where the complex exponent $\nu_{m}$ of the powers coincides with the order $\nu_{m}$ of the 'Riemann' differintegration, which is denoted by small $\mathrm{d}^{\nu} / \mathrm{dz} \mathrm{z}^{\nu}$, to distinguish from the capital $\mathrm{D}^{\nu} / \mathrm{Dz}{ }^{\nu}$, used for the 'Liouville' differintegration in the F.D.E. (2.1) of Standard I. The 'Riemann' differintegration applies to a function with a branch-point of exponent $\mu$, viz. to $F(z)=z^{\mu} f(z)$, where $f(z)$ is analytic, and is specififed (Lavoie and Osler and Tremblay [28]; Campos [25]) by: (1) a definite integral along the line $0, z$ :

$$
d^{\nu}\left\{z^{\mu} f(z)\right\} / d z^{\nu}=\{\Gamma(-\nu)\}^{-1} \int_{0}^{z}(\zeta-z)^{-\nu-1} f(\zeta) \zeta^{\mu} d \zeta,
$$

if $1+\operatorname{Re}(\mu)>0>\operatorname{Re}(\nu) ;$ (ii) a Pochhammer [29] double-loop integral,

$$
\begin{aligned}
& d^{\nu}\left\{z^{\mu} f(z)\right\} / d z^{\nu}=\left\{\Gamma(1+\nu) /\left(4 \pi e^{i \pi \mu} \sin (\pi \mu)\right)\right\} \\
& \int^{(z+, 0+, z-, 0-)}(\zeta-z)^{-\nu-1} f(\zeta) \zeta^{\mu} d \zeta,
\end{aligned}
$$

for complex $\mu, \nu$ other than $\mu$ integer and $\nu$ a negative integer. If all $\nu_{m}$ are positive integers, then $(7.1)$ is an Euler type 0.D.E. (Ince [30]), but if one or more $\nu_{m}$ are non-integral, then (7.1) with $(7.2)$ or $(7.3)$ is an intergrodifferential equation, of the generalized Volterra type, with power-type coefficients.

§8. SOLUTION IN TERMS OF BRANCHED VERSUS ANALYTIC FUNCTIONS.

The designation just given to the F.D.E., stems from the fact that the simplest instance, with $G(z)=0=v_{1}, v_{2} \equiv \nu, \lambda \equiv A_{1} / A_{2}, m=2$, is

$$
z^{\nu} d^{\nu} F / d z^{\nu}-\lambda F(z)=G(z)
$$

which, on account of (5.23), is a Volterra [21] type of integral equation (Hilbert \& Courant [31]), sometimes also designated (Whittaker and Watson [32]) a Fredholm equation of the second kind, and the case $\lambda=0$ is a Fredholm equation of $f$ irst kind. If all $\nu_{1}, \ldots, \nu_{m}$ are integers, it is well-known that the Euler linear equation (7.1) can be transformed into the type (2.1) with constant coefficients, via an exponential change of independent variable. This elementary transformation does not apply as well in the case of differintegrations with complex order $v$, since in this case, the rule of implicit differintegration (Campos [6])

$$
d^{\nu}\{F(z)\} / d\left\{e^{z}\right\}^{\nu}=\lim _{\zeta \rightarrow z} \frac{d^{\nu}}{d \zeta^{\nu}}\left\{F(\zeta)\left[\frac{e^{z}-e^{\zeta}}{z-\zeta}\right]^{\zeta-1} e^{\zeta}\right\}
$$

is not readily substituted in ( 8.1$)$. 
The difficulty in transforming the Euler type (Standard 11) fractional differential equation (7.1) into the type (Standard I) with constant coefficients (2.1), is rather fundamental, because the former uses the 'Riemann' differintegration (7.2), (7.3) and the latter the 'Liouville' differintegration (2.2), (2.3). Although the two systems of differintegration look superficially similar in the integrands, they use different paths in the complex plane (2.3), (7.3), i.e. the 'Liouville' system involves indefinite (2.2) and the 'Riemann' system involves definite (7.2) integrals. The two systems of differintegration are incompatible (Campos [25]) for non-integral $\nu$, because the Hankel path in (2.3) cannot be continuously deformed into the Pochhammer loop in (7.3), viz. the 'Liouville' system applies to analytic functions, whereas the 'Riemann' system applies to functions with one branch-point. It has been shown (Lavoie and Tremblay and osler [3]), that simultaneous application of the 'Liouville' [17] rule (3.2) and the 'Riemann' [33] rule of differintegration

$$
d^{\nu}\left\{z^{\mu}\right\} / d z^{\nu}=\{\Gamma(1+\mu) / \Gamma(1+\mu-\nu)\} z^{\mu-\nu},
$$

can lead to contradictions, if $\nu$ is not an integer.

The preceding remarks serve as a warning that the differintegration operators are more than just replacing integer for complex order of differentiation although some rules, such as (3.2) or (8.3), do work out that way; the theory of differintegration does have its subtle points, when compared with ordinary differentiation. We leave these matters to the references, and continue the presentation of results, which can be deduced in a very simple way, looking on the surface just like straightforward extensions of ordinary derivatives. The rules of differintegration have to be proved from the definitions of the integral operators like $(2.2),(2.3),(7.2),(7.3)$, and we are using only the simplest expressions holding for complex order v. Alternative methods of solution, like changes of variable, which hold for O.D.E.s, may fail to have simple extension to F.D.E.s, if the rules of differentiation have no straightforward and valid extension to differintegrations. Thus we proceed to solve F.D.Es ( 8.1 ) and (7.1), starting with the homogeneous case, and using the rule ( 8.3 ), which holds (Campos [25]) for all complex $\mu, \nu$ other than $\mu$ a negative integer. Since the actual calculations are very simple, and somewhat analogous to those in Part $I$, they are mentioned briefly in the following sections.

§9. ROOTS OF EQUATIONS INVOLVING GAMMA FUNCTIONS.

The homogeneous case of $(8.1)$, viz.

$$
z^{\nu} d^{\nu} F / d z^{\nu}=\lambda F(z)
$$

has a power type solution (9.1a)

$$
F(z)=z^{a}, \Gamma(1+a) / \Gamma(1+a-v)=\lambda,
$$

where a satisfies (9.2b), which was deduced using ( 8.3$)$. Thus the exponent a of (9.2a) is a root of (9.2b), which, for $v=n$ a positive integer, is a polynomial equation of degree $n, v i z$. 


$$
\lambda=\Gamma(1+a) / \Gamma(1+a-n)=a(a-1) \ldots(a-n+1),
$$

The $n$ roots $a_{k}$ with $k=1, \ldots, n$ specify the $n$ particular integrals of (9.1), which is, in this case, the original Euler equation. If $v$ is non-integral, the values of a are roots of the equation (9.2b) involving Gamma functions. Similarly, the general homogeneous fractional differential equation (7.1) of Standard II, has particular integrals of the form (9.2a), where a is a root of the discriminant equation

$$
0=P(a) \equiv \Gamma(1+a) \sum_{m=1}^{M}\left\{A_{m} / \Gamma\left(1+a-\nu_{m}\right)\right\},
$$

which is: (1) a polynomial, if all exponents $\nu_{1}, \ldots, \nu_{\mathrm{m}}$ are integers; (ii) a transcendental equation involving Gamma functions if at least one $\nu_{m}$ for $1<m<M$ is non-integral.

If the discriminant equation (9.4) has only simple roots $a_{k}$, with $k=\alpha, \ldots, \beta$, then the corresponding particular integrals $(9.2 a)$ are linearly independent, and the general integral is a linear combination

$$
F(z)=\sum_{k=\alpha}^{\beta} C_{k} F_{k}(z)=\sum_{k=\alpha}^{\beta} C_{k} z^{a_{k}},
$$

with constant coefficients $c_{k}$. If the root $a_{k}$ is of multiplicity $s$, then a procedure similar to the derivation from $(4.8)$ to $(4.9)$, would show that there are s particular integrals :

$$
F_{k}^{l}(z)=\partial^{l}\left\{F_{k}(z)\right\} / \partial\left(a_{k}\right)^{l}=(\log z)^{l} z^{a_{k}},
$$

with $\ell=0, \ldots, 8-1$; the particular integrals (9.6) are linearly independent for different roots (distinct $a_{k}$ ) and different multipliciltes (distince $\ell$ ). Thus, if the discriminant equation (9.4) has roots $a_{k}$ with $k=\alpha, \ldots, \beta$, of multiplicities $s_{k}$ (4.9), the general integral of the homogeneous fractional differential equation of Standard II,

$$
\sum_{m=1}^{M} A_{m} z^{\nu_{m}} d^{\nu_{m}} F / d z^{\nu_{m}}=0
$$

Is given by

$$
F(z)=\sum_{k=\alpha}^{B} z^{a_{k}} \sum_{\ell=0}^{8_{k}-1} c_{k}^{\ell}(10 g z)^{\ell},
$$

where the $C_{k}^{l}$ are constants. In the case $s_{k}=1$ when all roots are $81 \mathrm{mp} 1 \mathrm{e},(9.8)$ simplifies to $(9.5)$ with $C_{k}^{0} \equiv C_{k}$.

As an example, the F.D.E. of standard II,

$$
z^{1 / 2} d^{1 / 2} F / d z^{1 / 2}-z^{-1 / 2} d^{-1 / 2} F / d z^{-1 / 2}=0,
$$

has discriminant equation

$$
0=P(a)=\{\Gamma(1+a) / \Gamma(1 / 2+a)\}\{(2 a-1) /(2 a+1)\}
$$


One root of $(9.10)$ is $a=1 / 2$, showing that $\sqrt{z}$ is a solution of the F.D.E. (9.9), as can be checked using ( 8.3$)$. Generally F.D.E.s are not reducible to 0.D.E.s but in the particular case (9.9), this is possible via the change of dependent variable (9.11a)

$$
H(z)=d^{-1 / 2} F / d z^{-1 / 2}, F(z)=d^{1 / 2} H / d z^{1 / 2},
$$

where $(9.11 \mathrm{~b})$ is the inverse of $(9.11 \mathrm{a})$, obtained using the identity $d^{\nu} d^{-\nu}=1=d^{-\nu} d^{\nu}$ proved elsewhere (Campos [6]). The change of variable (9.11a) transforms the F.D.E. (9.9) to the O.D.E. (9.11a):

$$
H(z)=z d H / d z, H(z)=a z, \quad(9.12 a, b)
$$

which has solution ( $9.12 \mathrm{~b})$; the latter corresponds, through the inverse change of varibale (9.12ab), to the solution $F(z) \sim \sqrt{z}$ of $(9.9)$ apart from an irrelevant constant factor.

§10. F.D.E. WITH POWER-TYPE COEFFICIENTS AND FORCING.

To conclude the study of Standard II, we consider the inhomogeneous fractional differential equation ( 7.1 ) with power-type forcing

$$
\sum_{m=1}^{M} A_{m} z^{\nu_{m}} d^{\nu_{m_{F}} / d z^{\nu_{m}}}=B z^{b} .
$$

A particular integral is the same complex power

$$
P(b) \neq 0 ; F_{0}(z)=\{B / P(b)\} z^{b} \text {, }
$$

where the coefficient involves the discriminant function (9.4), and it is assumed that $b$ is not one of its roots; if $b$ is a root of multiplicity $s$ of the discriminant function (9.4), then

$$
P(b)=P^{\prime}(b)=\ldots=P^{(s-1)}(b)=0 \neq P^{(s)}(b) ; F_{0}(z)=\left\{B / P^{(s)}(b)\right\}(\log z)^{s} z^{b} \text {, }
$$

the particular integral (10.2) is replaced by (10.3). The latter (10.3) is deduced by a procedure similar to that used in $\$ 5$, to derive (5.10) from $(5.5 a, b)$; in the case b is not a root of (9.4), the result (10.3) with $s=0$ coincides with (10.2). The complete integral of the inhomogeneous F.D.E. (10.1) of Standard II consists of the sum of the particular integral (10.2) or (10.3), with the general integral (9.5) or (9.8) of the homogeneous F.D.E. $(9.4)$.

As an example we consider the inhomogeneous F.D.E.

$$
z^{1 / 2} d^{1 / 2} D / d z^{1 / 2}-z^{-1 / 2} d^{-1 / 2} F / d z^{-1 / 2}=\sqrt{\pi} z^{b}
$$

A particular integral is $(10.2)$ with $(9.10)$ :

$$
b \neq 1 / 2: F_{0}(z)=\{\Gamma(1 / 2+b) / \Gamma(1+b)\}\{(2 b+1) /(2 b-1)\} \sqrt{\pi} z^{b},
$$

provided that $b \neq 1 / 2$. If $b=1 / 2$, we evaluate the derivative of $(9.4)$ :

$$
\begin{aligned}
& P(a)=\Gamma(1+a)\{1 / \Gamma(1 / 2+a)-1 / \Gamma(3 / 2+a)\} \\
& P^{\prime}(a)=\{\Gamma(1+a) / \Gamma(1 / 2+a)\}\{\psi(1+a)-\psi(1 / 2+a)\}
\end{aligned}
$$




$$
-\{\Gamma(1+a) / \Gamma(3 / 2+a)\}\{\psi(1+a)-\psi(3 / 2+a)\}
$$

where $\psi(z) \equiv \Gamma^{\prime}(z) / \Gamma(z)$ denotes the Digamma function (Copson [34]). Since $P^{\prime}(1 / 2)=\sqrt{\pi} / 2$, the particular integral $(10.3)$ with $s=1, b=1 / 2, B=\sqrt{\pi}$ is given by

$$
b=1 / 2: F_{0}(z)=2 \sqrt{z} \log z \text {. }
$$

The general integral of the F.D.E. (10.4) is obtained by adding to (10.5) or (10.7), the expression $C \sqrt{z}$, where $C$ is a constant.

The change of variable (9.11a) transforms the F.D.E. (10.4) into an 0.D.E.:

$$
z d H / d z-H(z)=\sqrt{\pi} z^{b+1 / 2} \text {. }
$$

The particular integral of $(10.8)$ is:

$$
H_{0}(z)= \begin{cases}\sqrt{\pi}(b-1 / 2)^{-1} z^{b+1 / 2} & \text { if } b \neq 1 / 2, \\ \sqrt{\pi} z \log z & \text { if } b=1 / 2 .\end{cases}
$$

Substitution of $(10.9 a, b)$ into $(9.11 b)$, and use of (8.3), yields respectively (10.5) and (10.7). It could also be checked, by direct substitution, that (10.5) satisfies $(10.4)$ for $b \neq 1 / 2$, and $(10.7)$ satisfies $(10.4)$ for $b=1 / 2$. The former proof uses $(8.3)$, whereas the latter uses:

$$
\begin{aligned}
& d^{\nu}\left\{z^{\mu} \log z\right\} / d z^{\nu}=\{\Gamma(1+\mu) / \Gamma(1+\mu-\nu)\} \\
& z^{\mu-\nu}\{\log z+\psi(1+\mu)-\psi(1+\mu-\nu)\},
\end{aligned}
$$

with $\mu=1 / 2, \nu= \pm 1 / 2$; the formula (10.10) follows from (8.3) by parametric differintegration with regard to $\mu$, on account of the uniform convergence in $\mu$.

\$11. PROPERTIES ON NON-LINEAR F.D.E.S OF STANDARD III.

We have so far considered two classes (Standard I and II) of linear F.D.E.s and conclude with some results on non-linear F.D.E.s of Standard III:

$$
z^{\mu} d^{\nu} F / d z^{\nu}=\lambda\{F(z)\}^{b}
$$

The linear case $b=1$, includes the Standard $I$ (3.1) for $\mu=0$, and the Standard II (9.1) for $\mu=v$. We cannot expect the very simple methods used in the present paper to go very far towards solving a non-linear F.D.E. such as (11.1); thus we consider only the question of existence of a power type solution (9.2a), which, when substituted into (11.1) yields:

$$
\{\Gamma(1+a) / \Gamma(1+a-\nu)\} z^{a+\mu-\nu}=\lambda z^{a b}
$$


the equation (11.2) implies two identities:

$$
\lambda=\Gamma(1+a) / \Gamma(1+a-v), a=(\mu-\nu) /(b-1), \quad \text { (11.3a,b) }
$$

among which a can be eliminated:

$$
\Gamma(1+(\mu-v) /(b-1))=\lambda \Gamma(1+(\mu-b v) /(b-1)) .
$$

It follows that the non-linear F.D.E. (11.1) has a power-type solution (9.2a), if and only if the parameters $\mu, \nu, \lambda, b$ satisfy (11.4); in the latter case the exponent is given by $(11.3 \mathrm{~b})$.

For example, the non-linear fractional differential equation

$$
z^{1 / 2} d^{-1 / 2} F / d z^{-1 / 2}=\lambda\{F(z)\}^{2}
$$

has power type solution, for $\lambda$ given by (11.6a)

$$
\lambda=\Gamma(2) / \Gamma(5 / 2)=4 /(3 \sqrt{\pi}), a=1,
$$

with exponent (11.6b). Thus $z$ is a solution of the non-1inear F.D.E.

$$
z^{1 / 2} d^{-1 / 2} F / d z^{-1 / 2}=\{4 /(3 \sqrt{\pi})\}\{F(z)\}^{2}
$$

as can be checked using (8.3).

The equation (11.1) can be generalized to non-linear fractional differential equations of Standard III:

$$
\sum_{m=1}^{M} A_{m} z^{\mu_{m}} d^{\nu_{m}} m_{F / d z}{ }^{\nu}=\{F(z)\}^{b},
$$

which is linear for $b=1$ or $b=0$; in the latter case it reduces to Standard I (2.1) for $\mu_{1}=\ldots=\mu_{M}=0$, and to standard $\operatorname{II}(7.1)$ for $\mu_{m}=\nu_{m}$. The non-linear F.D.E. (11.8) is homogeneous, has power type solutions (9.2a) if the identity

$$
\sum_{m=1}^{M} A_{m}\left\{\Gamma(1+a) / \Gamma\left(1+a-\nu_{m}\right)\right\} z^{a+\mu_{m}-\nu_{m}}=\lambda z^{a b},
$$

1s satisfied. This implies a set of $M+1$ equations:

$$
\begin{aligned}
& a(b-1)=\mu_{1}-\nu_{1}=\ldots=\mu_{m}-\nu_{m}, \\
& 1 / \Gamma(1+a)=\sum_{m=1}^{M} A_{m} / \Gamma\left(1+a-\nu_{m}\right),
\end{aligned}
$$

from which a can be eliminated, leading to a set of $M$ restrictions on the $3 M+1$ parameters $A_{m}, \mu_{m}, \nu_{m}, b$, which have to be satisfied, in order that (11.8) has a solution 
of the form (9.2a). Less simple properties of differintegrations (Campos [36, 37]) may also have applications to F.D.E.S.

\section{REFERENCES}

1. ROSS, B., Fractional Calculus and Applications, Conf. Proceed, New Haven, Springer-Verlag, 1974 .

2. OldHAM, K.B. and SPANIER, J., Fractional Calculus, Academic Press, 1974.

3. LAVOIE, J.L., OSLER, T.J., TREMBLAY, R., Fractional Derivatives and Special Functions, SIAM Review 18 (1976), 240-268.

4. MCBRIDE, A.C., Fractional Calculus and Integral Transforms of Generalized Functions, Pitman, 1979.

5. NISHIMOTO, K., Fractional Calculus, Descartes Press, Koryama, Japan, 2 Vols. 1984-6.

6. CAMPOS, L.M.B.C., On a Concept of Derivative of Complex Order with Application to Special Functions, IMA Journ. App1. Maths. 33 (1984), 109-133.

7. MCBRIDE, A.C. and ROACH, G.F., Fractional Calculus, Pitman, 1986.

8. WEINSTEIN, A., Generalized Axially Symmetric Potential Theory, Bull. Amer. Math. Soc. $59(1953), 20-38$.

9. ERDELYI, A., An Integral Equation Involving Legendre Polynomials, J. SIAM 12 (1964), 15-30.

10. ERDELYI, A., Axially-Symmetric Potentials and Fractional Integration, J. SIAM 13 (1965), 216-228.

11. OLDHAM, K.B. and SPANIER, J., Replacement of Fick's Laws by a Formulation Involving Semidifferentiation, J. Electroanal. Chem. Interf. Electrochem. 26 (1970), 331-335.

12. LIGHTHILL, M.J., Waves in Fluids, Cambridge U.P., 1978.

13. CAMPOS, L.M.B.C., On waves in gases: Acoustics of jets, turbulence and ducts, Rev. Mod. Phys. 58 (1986), 117-182.

14. MARSTON, P.L., Half-order Derivative of a Sine-wave Burst: Applications to TwoDimensional Radiation, Photoacoustics, and Focused Scattering from Spheres and a Torus, J. Acoust. Soc. Am. 76 (1984), 291-295.

15. CAMPOS, L.M.B.C. and SANTOS, A.J.P., On Longitudinal Oscillations of a Tapered Visco-Elastic bar J. Sound Vib. 126 (1988), 109-125.

16. OLDHAM, K.B. and SPANIER, J., General Solution of the Diffusion Equation for SemiInfinite Geometries, J. Math. Anal. Appl. 39 (1972), 655-665.

17. LIOUVIlle, J., Mémoire sur le Calcul des Differentielles á Indices Quelconques. J. Ec. Polyt. $13(1832), 71-162$.

18. LIOUVILLE, J., Sur le Changement de Variable Independant dans le Calcul des Differentielles á Indices Quelconques, J. Ec. Polyt. 24 (1835), 15-54.

19. LIOUVILLE, J., Sur l'intégration des Equations Differentielles á Indices Fractionaires, J. Ec. Polyt. 25 (1837), 58-84.

20. SNEDDON, I.N., The Use of Integral Transforms, McGraw-Hil1, 1974. 
21. VOLTERRA, V., Theory of Functionals, Blackie, repr. Dover, 1959.

22. DUARTE, R.T., A new technique for the analysis of strong ground vibrations and the quantification of earthquake actions, Proc. 7th Europ. Conf. Earthq. Eng., Athens, 1983.

23. DUARTE, R.T., A Generalized Damping Motion, Lab. Nac. Eng. Civil report, Lisbon, 1984.

24. FORSYTH, A.R., Treatise of Differential Equations, MacMillan, 6th ed., 1885.

25. CAMPOS, L.M.B.C., On Rules of Derivation with Complex Order of Analytic and Branched Functions, Portug. Mathem. 43 (1985), 347-376.

26. HANKEL, H., Die Euler'schen Integrale bei Unbeschrankter Variabilitat des Argumentes, Zeft. Math. Phys. 9 (1864), 7-21.

27. Liouville, J., Sur 1'integration de 1 'equation $\left(m x^{2}+n x+p\right) d^{2} y / d x^{2}+(q x+r)$ $\mathrm{dy} / \mathrm{dx}+\mathrm{sy}=\mathrm{q}$ á $\mathrm{l}^{\prime}$ aide de Differentielles á Indices Quelconques. J. Ec. Polyt. 21 (1832b), 163-189.

28. LAVOIE, J.L., TREMBLAY, R. and OSLER, T.J., Fundamental Properties of Fractional Derivatives via Pochhammer Integrals, In Ross, 1974, 323-356.

29. POCHнAMmER, L., Ueber ein Integral mit Doppeltem Umlauf, Math. Ann. 75 (1890), 470-494.

30. INCE, E.L., Ordinary Differential Equations, MacMillan, repr. Dover 1954.

31. COURANT, R. and HILBERT, D., Methods of Mathematical Physics, Interscience, 2 vols, (1953).

32. WHITTAKER. E.T. and WATSON, G.N., Course of Modern Analysis, Cambridge U.P., 6 th ed. 1927.

33. RIEMANN, B., Versuch elner allgemeinen Auf fassung der Integration und Differentiation, Ges. Werke (1847), 331-344.

34. COPSON, E.T., Functions of a Complex Variable, Oxford U.P, (1935).

35. CAMPOS, L.M.B.C., On a Systematic Approach to Some Properties of Special Functions, IMA Journ. Appl. Maths. 36 (1986), 191-206.

36. CAMPOS, L.M.B.C., On a Branch-point Operator and Anihilation of Differintegrations, SIAM J. Math. Anal. 20 (1989), 439-453.

37. CAMPOS, L.M.B.C., On a Generalized Mittag-Leffler Theorem and Implicit Differintegration, SIAM J. Math. Ana1. 20 (1989), 454-467. 


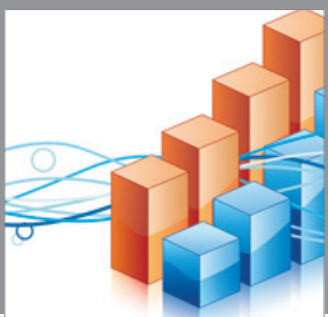

Advances in

Operations Research

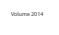

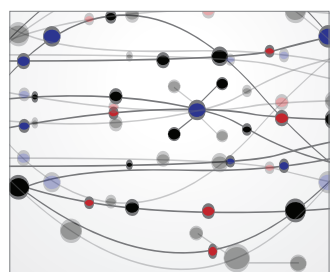

\section{The Scientific} World Journal
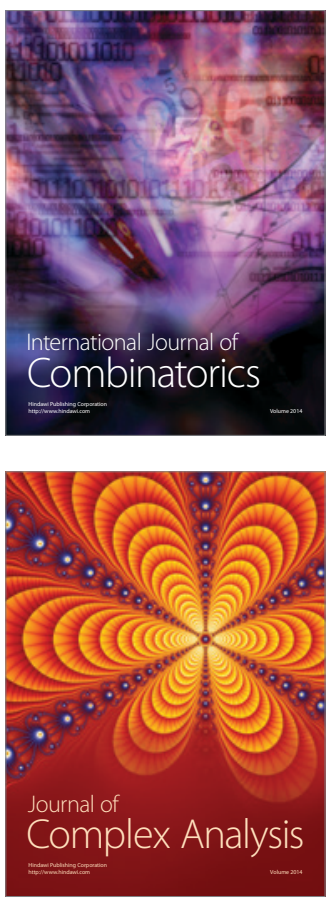

International Journal of

Mathematics and

Mathematical

Sciences
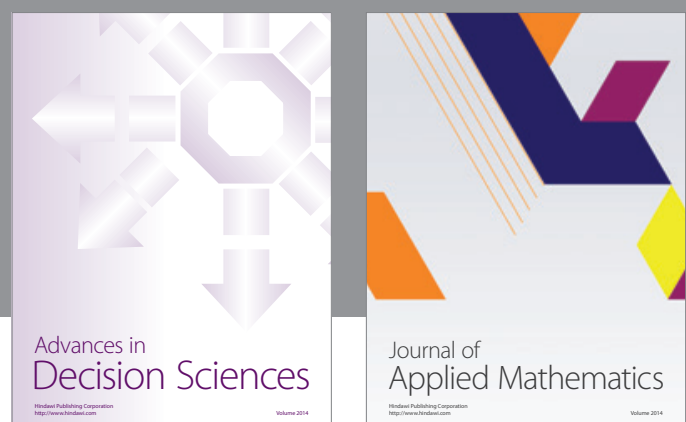

Journal of

Applied Mathematics
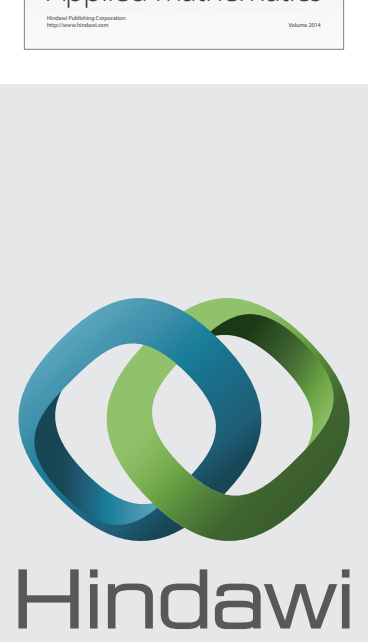

Submit your manuscripts at http://www.hindawi.com
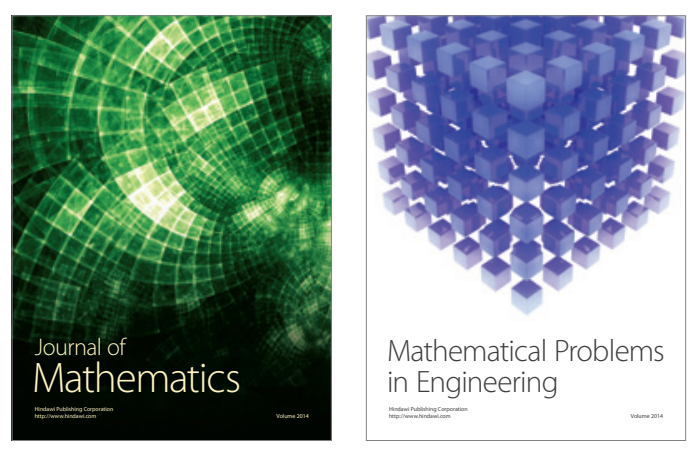

Mathematical Problems in Engineering
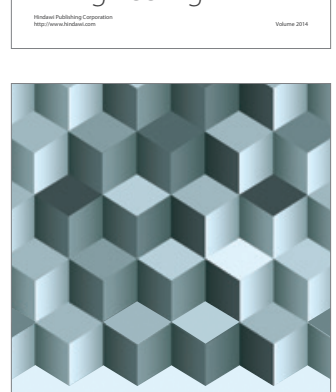

Journal of

Function Spaces
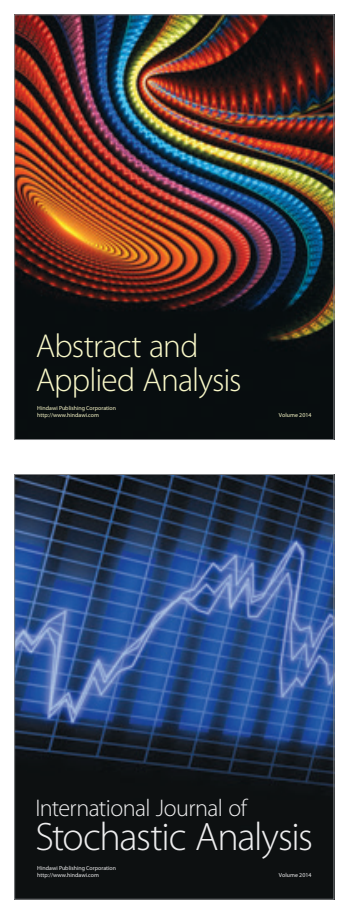

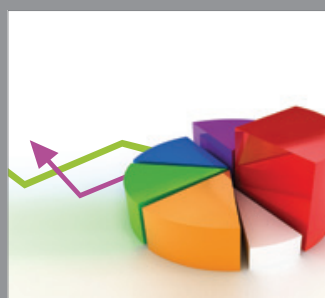

ournal of

Probability and Statistics

Promensencen
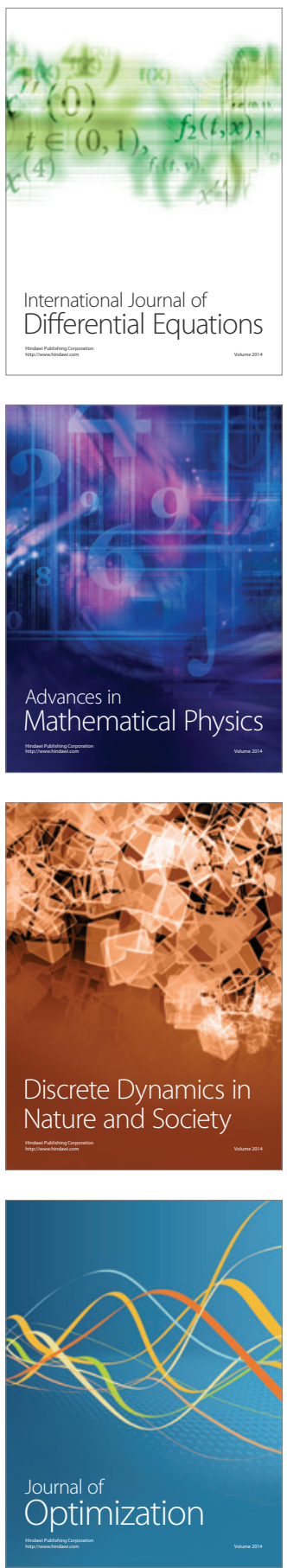